\title{
Analysis and compensation of workpiece errors in turning
}

\author{
XIAOLI LI $\dagger$ and R. DU $\star^{*}$
}

A new method for workpiece error analysis and compensation in turning is introduced. It is known that the workpiece error consists of two parts: machine tool error (including the geometric error and thermal-induced error) and cuttinginduced error. The geometric error of the machine tool is independent on machining operation and, hence, can be measured off-line using a fine-touch sensor with a Q-setter (FTS-Q) (also called quick touch setter). The thermal error of the machine tool is dependent on cutting speed, feed, machining time and environmental temperature. It can be estimated using a radius basis function (RBF) artificial neural network (ANN). The cutting-induced error plays a dominant role and can be estimated based on the cutting condition (speed, feed and depth of cut) and the motor currents (main spindle motor current and feed spindle motor current) using a two-stage RBF ANN. Based on the estimated error, the compensation can be done by overwriting the $\mathrm{CNC}$ code on-line. Experimental results indicate that the new method can reduce the workpiece error by as much as $75 \%$ (average workpiece error is reduced to $8 \mu \mathrm{m}$ from $14 \mu \mathrm{m}$ ). The new method is also easy to implement in the shop floor.

\section{Introduction}

In recent years, a great progress has been made in precision machining. Nowadays, it is possible to make precision parts with submicron or even nanometre accuracy, which paves the way for many applications in biomedical, electronic or aerospace engineering. However, a number of problems remain unsolved. For instance, the hardness of the cutting tools (e.g. diamond or diamond-coated carbide tools) and the workpiece (e.g. titanium alloys, metal-matrix composites, etc.) pose a major limitation on the accuracy of the machined parts and tool life (Asao et al. 1992). More importantly, to achieve high accuracy, ultra-precision machine tools are needed, which are very expensive. To improve the machining accuracy further without significant capital investment, real-time error compensation based on sensing and control techniques have been studied (Veldhuis and Elbestawi 1995, Yuan and Ni 1998, Liu and Venuvinod 1999).

It is known that a machining process consists of four parts: (1) the machine tool structure including the motor and spindle, (2) the tables of the machine tool and the driving system, (3) the workpiece, and (4) the cutting process. During the machining operations, various errors may occur including (Chen et al. 1998), as follows.

Revision received July 2001.

$\dagger$ Department of Manufacturing Engineering, City University of Hong Kong, Tat Chee Avenue, Kowloon, Hong Kong.

tDepartment of Automation and Computer Aided Engineering, The Chinese University of Hong Kong, Shatin, N.T., Hong Kong.

* To whom correspondence should be addressed. e-mail: rdu@acae.cuhk.edu.hk 
- Machine tool movement errors such as the errors caused by the dynamics of the driving system and the numerical truncation of the CNC.

- Machine tool structural errors such as thermal expansion errors and geometric errors.

- Cutting force-induced errors, such as machine tool deflection, cutter deflection, workpiece deflection, thermal expansion, tool wear and chatter.

These errors combined cause the dimensional error of the machined workpiece, called the workpiece error. In the present study, the workpiece error $(\delta)$, is decomposed into three parts: the geometric error of the machine tool $\left(\delta_{\mathrm{G}}\right)$, the thermalinduced error of the machine tool $\left(\delta_{\mathrm{T}}\right)$ and the cutting force-induced error $\left(\delta_{\mathrm{F}}\right)$. The first two errors are related to the machine tool while the last one is dependent mainly on the cutting.

The geometric error of the machine tool is attributed to the inaccuracy of the machine tool and the cutter. In modern machine tools, this can be limited to the micron level or less. In general, the thermal error may result from the mechanical friction as the machine tool moves, and the heat generated by the cutting process. In Veldhuis and Elbestawi (1995), the distribution of the thermal errors is estimated using an artificial neural network (ANN). Bryan (1990) proposed that the spindle thermal drift is the dominant source of thermal-induce d errors.

The cutting force-induced error is the dominant error source when turning small workpieces or in hard turning. Yang et al. (1997) decomposed this error into ten different components. They subsequently developed a real-time error compensation system on a CNC turning centre using an independent computer controller. In Kops et al. $(1993,1994)$, the cutting force-induced error was converted to workpiece deflection. Then, an analytical formula was developed to correlate the deflection and the depth of cut. However, only the radial component of the cutting force was considered. In Phan et al. (1999), a new model was proposed for bar turning that included all cutting force components (radial, axial and tangential), but the model was rather complicated. It is interesting to note that all the existing methods require cutting force measurement. Consequently, their implementations could be costly.

This paper introduces a new method for workpiece error analysis, measurement, prediction and compensation. The paper consists of five sections. Section 2 describes the experimental set-up for measuring the workpiece error. In particular, it presents a workpiece error measurement system using a fine touch sensor and a Q-setter (FTS-Q) (also called quick touch setter). In Section 3, the sources of the workpiece errors are studied and these errors are estimated using various means including hybrid radius based function (RBF) ANN. Section 4 presents the experiment results. Finally, Section 5 has conclusions.

\section{Measurement set-up}

The measurement set-up was established on a CNC turning centre (model HITEC TURN 20SII by Seiki-Seicos). According to the literature, to measure the workpiece dimension, a number of contact sensors have been developed, such as the touch trigger probe MP3 (Phan et al. 1999). While these contact sensors are accurate, their applications have been limited because of their complexity, manufacturing cost and maintenance cost. Recently, Ostafiev et al. (1991) and Ostafiev and Venuvinod (1997) developed a sensing system called fine touch sensor (FTS). One of the most 


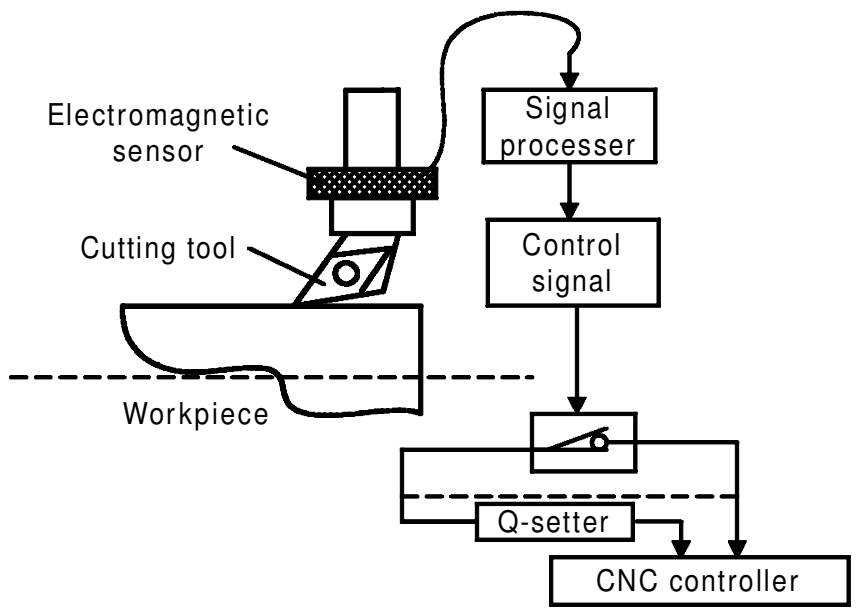

Figure 1. Illustration of the FTP-Q sensing system.

important features of FTS is that it uses the cutting tool tip as a contact probe. As shown in figure 1, the principle of FTS is rather simple. When the tool touches the workpiece, a closed-loop electric circuit is formed changing the electromagnetic field. Consequently, an impulse signal is generated. FTS is capable of achieving the measurement accuracy comparable with that of the best touch trigger probes (about $0.01 \mu \mathrm{m})$. Yet, its construction and operation are relatively simple, and its manufacturing cost and maintenance cost are relatively low. Here, such a FTS was used to measure the workpiece diameter on-line.

Figure 2 illustrates the set-up for measuring the workpiece diameter. As shown, an FTS is mounted on the CNC turning centre. Like many modern turning machining centres, the HITECT TURN 20SII machine is equipped with a 'Quick Tool

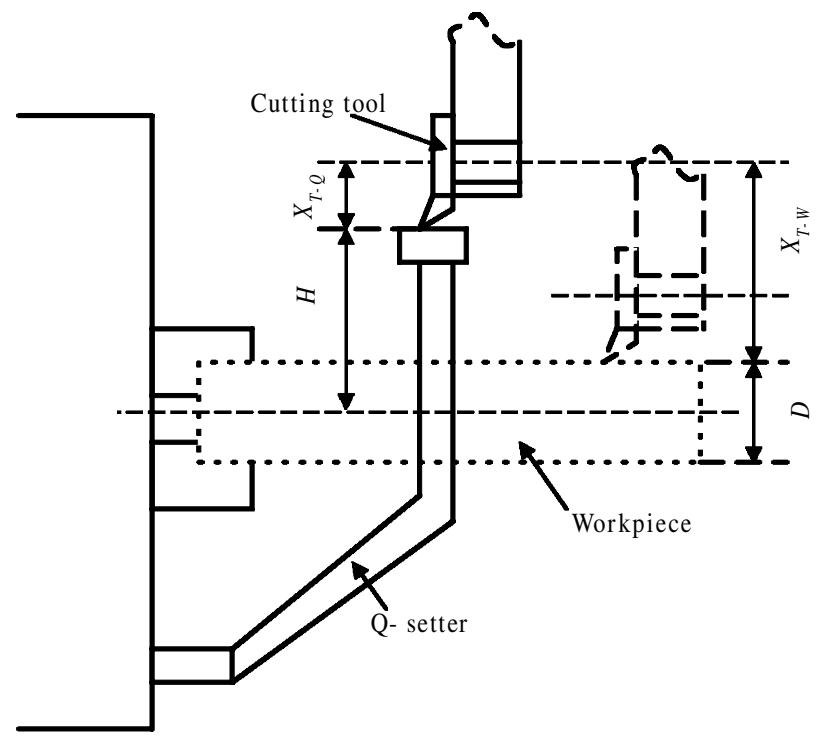

Figure 2. Illustration of the experiment setup. 
Setter' or 'Q-setter', which is designed to facilitate the tool changing. When the cutter touches it, an impulse is generated and sent to the CNC controller. This impulse signal can be used to program an offset corresponding to the workpiece. In our measurement set-up, it is used together with FTS forming the FTS-Q sensing system. During the measurement, first the cutter is moved to touch the Q-setter, at which time a tool offset, $X_{\mathrm{T}-\mathrm{Q}}$, is obtained. Then, when the cutting tool touches the workpiece, another signal is generated that stops the machine tool. At the same time, a tool offset, $X_{\mathrm{T}-\mathrm{w}}$, is generated. From figure 2, it is seen that the diameter of the workpiece, $D_{\text {om }}$, is:

$$
D_{\text {om }}=2 \times\left(H+\left|X_{\mathrm{T}-\mathrm{Q}}\right|-\left|X_{\mathrm{T}-\mathrm{W}}\right|\right),
$$

where $H$ is the distance from the centre of the Q-setter to the centre of the spindle in the $x$-axis, which is provided by the machine tool manufacturer. For the HITECT TURN 20SII machine, $H=85.356 \mathrm{~mm}$.

\section{Workpiece error analysis and compensation}

As pointed out above, the workpiece errors may be attributed to various sources. For convenience, the following notations are used.

$D_{\text {des }}$ desired (or designed) dimension of the workpiece,

$D_{\text {omw }}$ dimension measured using the on-machine measurement device (FTS-Q) immediately after the machining operation (the subscript 'omw' indicates it is on the machine and warm),

$D_{\text {omc }}$ dimension measured using FTS-Q after the machine has cooled down (the subscript 'omc' indicates it is on the machine and cold), and

$D_{\mathrm{pp}} \quad$ dimension measured using a coordinate measurement machine (CMM) (post-process measurement).

Note that the desired dimension of the workpiece, $D_{\text {des }}$, is known before cutting and the measurement error of the CMM, $D_{\mathrm{pp}}$, is very smaller. Hence, the total workpiece error is approximately:

$$
\delta_{\mathrm{Tot}}=\frac{D_{\mathrm{pp}}-D_{\mathrm{des}}}{2} \approx \delta_{\mathrm{G}}+\delta_{\mathrm{F}}+\delta_{\mathrm{T}} .
$$

\subsection{Geometry errors}

The geometric error of the machine tool can be obtained based on the measurements from the FTS-Q. First, the diameters of a precision ground test artefact were measured at 10 different positions, $20 \mathrm{~mm}$ apart from each other, by a CMM, and their readings were denoted as $D_{\mathrm{pp}}(i), i=1,2, \ldots, 10$. Second, the test artefact was mounted on the machine tool and its diameters were measured in the same manner by using the FTS-Q, and their readings were denoted as $D_{\text {omc }}(i)$, $i=1,2, \ldots, 10$. Accordingly, the geometric error was found using:

$$
\delta_{\mathrm{G}}(i)=\frac{\left(D_{\mathrm{pp}}(i)-D_{\mathrm{omc}}(i)\right)}{2} .
$$

Figure 3 summarizes the experimental results. It is seen that the average geometric error ranges from 7 to $14 \mu \mathrm{m}$ depending on the diameter of the workpiece. In general, the larger the workpiece, the larger the geometry error. This is perhaps attributed to 


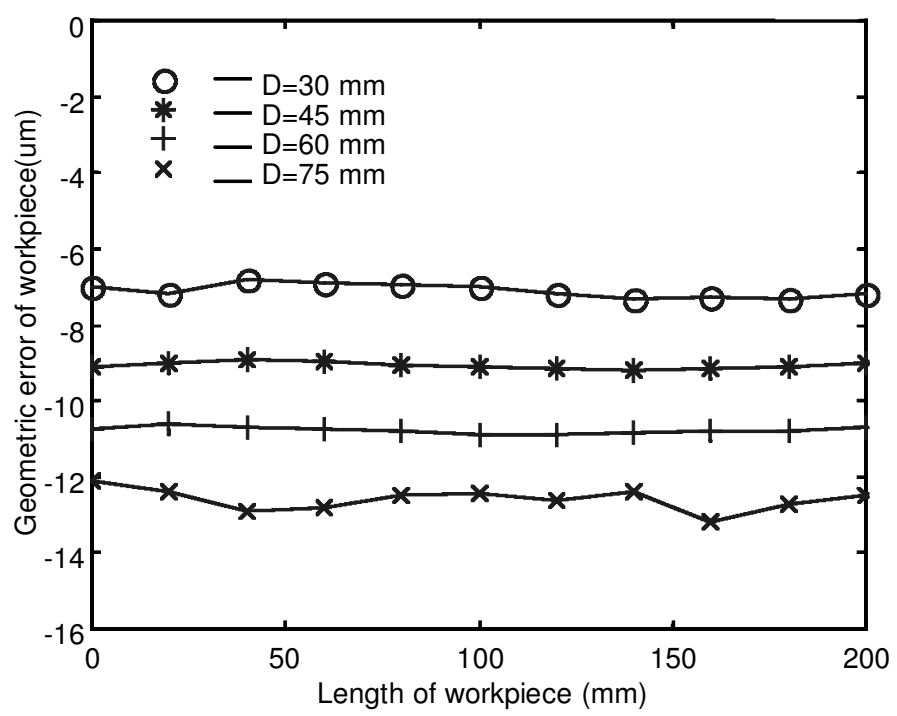

Figure 3. The geometric errors of the workpiece of a CNC turning center.

the linear error of the table in the $x$-direction. The measurement error may also play a role. Furthermore, applying linear regression, it was found that the geometric error can be modelled using:

$$
\delta_{\mathrm{G}}(\phi)=-0.121 \phi-3.519,
$$

where $\phi$ is the diameter of the workpiece $(\mathrm{mm})$.

\subsection{Thermal-induced errors}

In general, thermal error may result from the mechanical friction as the machine tool moves as well as the heat generated by the cutting process. This paper focuses on the former since the latter can be considered as a part of the cutting force-induced error, which will be dealt with below. A machine tool has a number of moving parts and the corresponding thermal errors can be decomposed into radial, axial and tilt components. In bar turning, however, the dimensional deviation of the workpiece is mainly effected by the radial thermal drift and, hence, the radial thermal drift is considered as the main source of the error.

The radial thermal drift $\left(d_{\text {radial }}\right)$ can be obtained based on the measurements from the FTS-Q. The schematic set-up for the measurement is depicted in figure 4. First, the diameters of the workpiece at points $A$ and $B$ were measured using the FTS-Q sensing system immediately after the machining operation (at the worm state), their readings are denoted as $D_{\text {omw }}(\mathrm{A})$ and $D_{\text {omw }}(\mathrm{B})$ respectively. Next, the diameter of the workpiece at the same points was measured using the FTS-Q sensing system when the workpiece cooled down and the readings were denoted as $D_{\text {omc }}(\mathrm{A})$ and $D_{\text {omc }}(\mathrm{B})$ respectively. Accordingly, the radial thermal drift can be found using:

$$
d_{\text {radial }}(A)=\frac{D_{\text {omw }}(A)-D_{\text {omc }}(A)}{2} \quad \text { and } \quad d_{\text {radial }}(B)=\frac{D_{\text {omw }}(B)-D_{\text {omc }}(B)}{2} .
$$

To measure the thermal-induced error without waiting for the machine to cool down is much more difficult. It is known that the thermal-induced error is dependent on a 


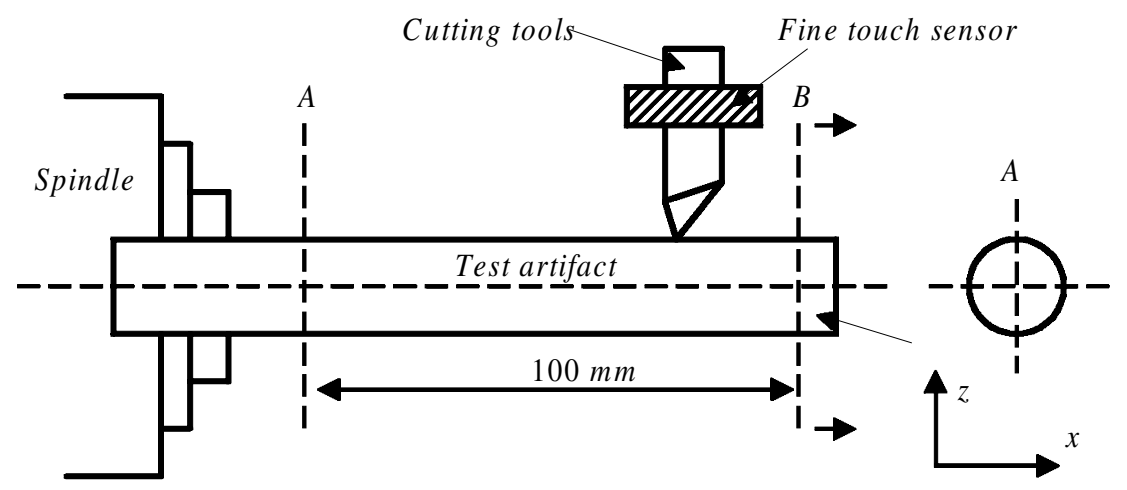

Figure 4. Illustration of the measurement of the thermal error using FTS-Q system.

number of factors such as the spindle speed, the machine tool operating time and the environmental temperature. To estimate the thermal-induced error, a hybrid RBF ANN (see appendix A) was used. As shown in figure 5, the inputs to the ANN include the following.

- Spindle speed: based on the fact that as the spindle rotates, the temperature at the bearings raises owing to friction, Also, the spindle motor generates heat.

- Feed: as the machine tool table moves, the friction between guide-ways and ball screw generates heat.

- Elapsed time since the machine tool started: in general, the temperature increases as the machining time increases. However, it usually stabilizes at a steady-state after some time.

- Environmental temperature of the machine tool.

The output of the network is the radial thermal drift. The model was trained off-line from the experiment data, and then used for on-line prediction of thermal-induced error. For the details of the model, see Li (2000).

\subsection{Cutting force-induce d errors}

Cutting force-induced error plays a dominant role. In practice, this error may result from various sources, such as the deflection of the machine tool (including the

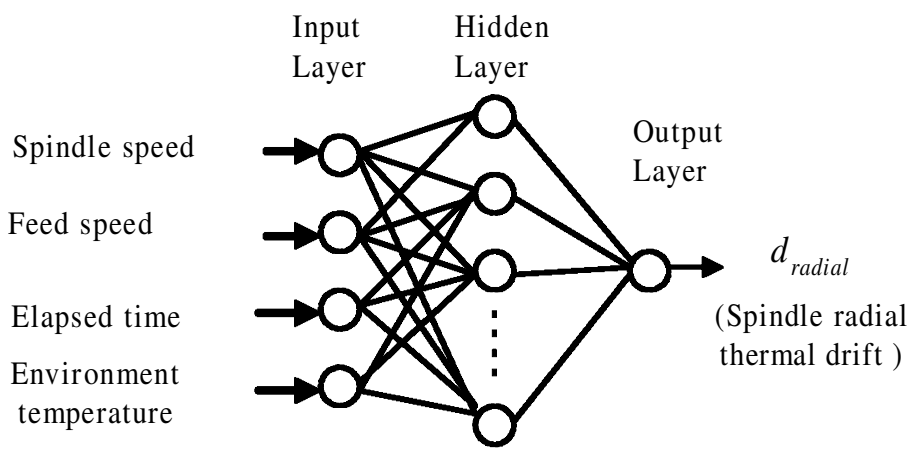

Figure 5. The hybrid RBS neural network for thermal induced error. 
cutter), the deflection of the workpiece and the thermal expansion of the workpiece due to the cutting. It is rather difficult to measure the cutting force-induced error. However, based on equations (2) and (4), it can be computed as follows:

$$
\delta_{\mathrm{F}}=\frac{D_{\mathrm{pp}}-D_{\mathrm{des}}}{2}-\delta_{\mathrm{G}}-\delta_{\mathrm{T}}
$$

Unfortunately, equation (6) cannot be used for on-line measurement because $D_{\mathrm{pp}}$ will not be available.

In general, the cutting force-induced error is difficult to compute since it is dependent on many factors such as the stiffness of the machine tool and the cutter, the cutting forces, and the cutter geometry, to name just a few. However, it is generally believed that the error is proportional to the deflection of the workpiece, which is determined by the cutting forces and the length from the chuck, $z$. Here, the cutting force-induced error is estimated by using a hybrid RBF ANN (see appendix A). As shown in figure 6, the inputs of the ANN include the three cutting forces (the tangential force, $F_{\mathrm{t}}$, the axial force, $F_{\mathrm{a}}$, and the radial force, $F_{\mathrm{r}}$ ), as well as the length from the chuck. To simplify the calculation, the tangential cutting force, $F_{\mathrm{t}}$, and the part diameter, $D$, are combined into a single variable: the spindle torque $T=F_{\mathrm{t}} D$. The outputs of the network are the cutting force-induced error.

It is well known that cutting forces can be measured using dynamometers. However, dynamometers are usually rather expensive. Moreover, the use of dynamometers may reduce the stiffness of machine tools leading to chatter and/or additional dimensional errors (Stein and Huh 1991). Hence, many attempts have been made to estimate the cutting forces using the motor current (Stein et al. 1986, Altintas 1992, Lee et al. 1995, Chang et al. 1995, Kim and Kim 1996, Haber et al. 1998). Here, a new method is developed to estimate the cutting forces based on the motor current as described below.

\subsubsection{Tangential force, $\mathrm{F}_{t}$}

The tangential force is related to the power consumption of the main spindle motor. Consider the main spindle and motor as a system. In this system, the following equation holds ( $\mathrm{Li}$ 2001):

$$
J_{\mathrm{s}} \dot{\omega}_{\mathrm{s}}=K_{\mathrm{s}} I_{\mathrm{s}}-B_{\mathrm{s}} \omega_{\mathrm{s}}-\left(T_{\mathrm{sf} 0}+\Delta T_{\mathrm{sf}}+\Delta T_{\mathrm{sv}}+T_{\mathrm{s}}\right)
$$

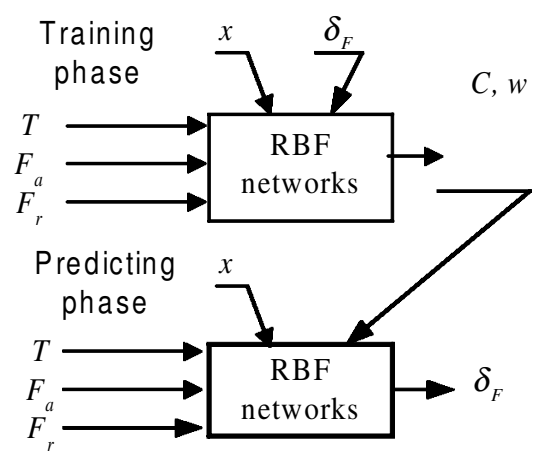

Figure 6. The model for cutting force induced errors. 
where $J_{\mathrm{s}}$ is the total equivalent inertia of spindle drive system; $\omega_{\mathrm{s}}$ is the spindle speed; $K_{\mathrm{s}}$ is the constant of the spindle motor; $I_{\mathrm{s}}$ is the armature current; $T_{\mathrm{s}}$ is the cutting torque due to tangential cutting force; $B_{\mathrm{s}}$ is the damping coefficient; $T_{\mathrm{sf}}$ is the dry friction of the spindle drive system; $T_{\mathrm{sf0}}$ is the coulomb friction torque at the idle state of the spindle system; and $\Delta T_{\mathrm{sf}}$ is additional coulomb friction torque of the spindle system due to cutting load. Note that the spindle speed, $\omega_{\mathrm{s}}$, can be calculated based on the frequency of the current signal (Li 2001):

$$
\omega_{\mathrm{s}}=2.8 \times f_{\mathrm{s}},
$$

where $f_{\mathrm{s}}$ is the frequency of current signal (in one of the three phases). On the other hand, the torque $\left(T_{\mathrm{s}}\right)$ is equal to the direct current (DC) current $\left(I_{\mathrm{s}}\right)$ multiplied by the motor torque constant $\left(K_{\mathrm{s}}\right)$ for the DC servo motor systems. For the alternative current (AC) servo motor systems with three-phase synchronous motors, the equivalent DC current can be found by converting the three-phase AC current using the following equation:

$$
I_{\mathrm{s}}=\sqrt{\frac{I_{U}^{2}+I_{V}^{2}+I_{W}^{2}}{3}} .
$$

Furthermore, the current consumption can be divided into three terms:

$$
I_{\mathrm{s}}=I_{\mathrm{s} 0}+\Delta I_{\mathrm{sf}}+\Delta I_{\mathrm{s}},
$$

where $I_{\mathrm{s} 0}$ is the idling current consumption, $\Delta I_{\mathrm{sf}}$ is the current consumed by the friction of spindle drive system and $\Delta I_{\mathrm{s}}$ is the current consumption due to cutting. In practice, $I_{\mathrm{so}}$ and $\Delta I_{\mathrm{sf}}$ can be measured without cutting. The cutting force-induced current, $\Delta I_{\mathrm{s}}$, is related to the cutting force, $F_{\mathrm{t}}$, the additional friction due to the cutting load and the angle acceleration of spindle system $\dot{\omega}_{\mathrm{s}}$. In summary, the following relationship exists:

$$
F_{\mathrm{t}}=F\left(\Delta I_{\mathrm{s}}, \omega_{\mathrm{s}}, \dot{\omega}_{\mathrm{s}}\right) .
$$

Equation (11) is a non-linear function and, hence, is difficult to solve. Here, a hybrid RBF neural network (see appendix A) is used to model this function. The inputs to the network include the cutting force-induced current $\Delta I_{\mathrm{s}}$, the spindle speed $\omega_{\mathrm{s}}$ and the spindle acceleration $\dot{\omega}_{\mathrm{s}}$. The output of the network is the estimated tangential cutting force, $F_{\mathrm{t}}$. This is illustrated in figure 7 .

\subsubsection{Axial force, $\mathrm{F}_{a}$}

The axial force is related to the feed system. In a CNC lathe, a typical feed system with an AC servo motor consists of several components: the cutting tool, the tool holder, the slide, the bearings, the ball screw, the feed box, and the AC motor. The inertia of the motor armature, feed box, ball screw and slide can be lumped together to form an equivalent inertia, $J_{\mathrm{a}}$. On the other hand, the damping of the feed box, the bearings, the ball screw and the slide can be lumped together to form an equivalent viscous damping, $B_{\mathrm{a}}$. The dry or Coulomb friction of the slide and other friction components can also be combined to form an equivalent friction torque $T_{\mathrm{af}}$. Finally, the axial cutting force component is related to the torque, $T_{\mathrm{a}}$, of the feed motor. If the effect of viscous and friction can be separated, the axial cutting force $F_{\mathrm{a}}$ can be 


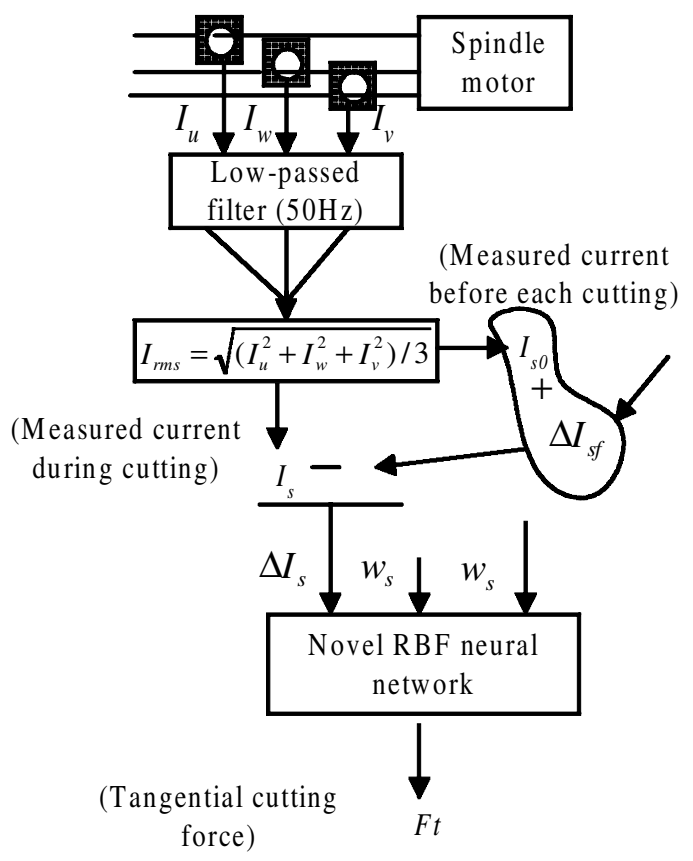

Figure 7. The hyper RBF neural network model for tangential cutting force.

estimated based on the axial motor current. In the steady cutting states, namely $\dot{\omega}_{\mathrm{a}}=0$, the following equation holds:

$$
K_{\mathrm{a}} I_{\mathrm{a}}=B_{\mathrm{a}} w_{\mathrm{a}}+T_{\mathrm{a}}+T_{\mathrm{af} 0}+\Delta T_{\mathrm{af}}+\Delta T_{\mathrm{av}},
$$

where $K_{\mathrm{a}}$ is the constant of the axial feed motor; $\omega_{\mathrm{a}}$ is the velocity of the axial feed motor; $T_{\mathrm{af} 0}$ is the Coulomb friction torque of axial feed system at the idle states; $\Delta T_{\text {af }}$ is the additional coulomb friction torque of axial feed system due to cutting; $\Delta T_{\mathrm{av}}$ is the additional viscous friction torque of axial feed system due to cutting load. The motor current, $I_{\mathrm{a}}$, consists of two parts: $I_{\mathrm{a}}=I_{\mathrm{a} 0}+\Delta I_{\mathrm{a}}, I_{\mathrm{a} 0}$ is the idling current and $\Delta I_{\mathrm{a}}$ is called the cutting current. The idling current is the current consumed at the idle state. It is independent of the cutting and hence, can be measured before cutting. The cutting current is used to overcome the axial cutting force, the additional friction caused by the cutting load, as well as the damping of the axial feed drive system $\left(B_{\mathrm{a}}\right)$. Since the additional friction is directly related to the axial feed cutting force and the damping of the feed drive system is dependant on the feed speed, $f_{\text {as }}$, the relationship of the axial cutting force can be expressed as follows:

$$
F_{\mathrm{a}}=F\left(\Delta I_{\mathrm{a}}, f_{\mathrm{as}}\right) .
$$

Note that the feed speed, $f_{\text {as }}$, is related to the frequency of the motor, $f_{\mathrm{a}}$ ( $\mathrm{Li} \mathrm{2001):}$

$$
f_{\text {as }}=0.3586 \times f_{\mathrm{a}} .
$$

Similar to tangential force, a hybrid RBF ANN is used to estimate the axial cutting force defined in equation (13). The inputs of the network include $\Delta I_{\mathrm{a}}$ and $f_{\text {as }}$, and the output of the network is the axial cutting force. This is shown in figure 8.

It should be pointed out that for the machine tools that use step motors, a similar RBF ANN may be used. 


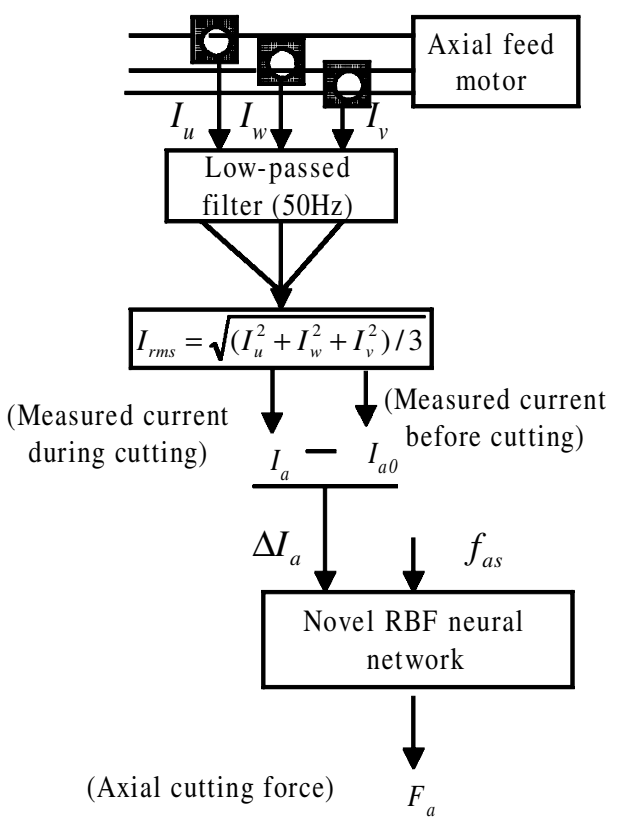

Figure 8 . The hyper RBS neural network model for axial cutting force.

\subsubsection{Radial cutting force}

In bar turning, the radial drive motor is not used. Hence, the radial cutting force cannot be estimated from the radial feed motor current. To overcome this problem, in this study, the radial cutting force was estimated using a semi-empirical mechanistic model. First, according to Endres et al. (1992) and Zhang et al. (1991), cutting forces are equal to the product of cutting pressures and uncut chip area. Hence, the specific normal cutting force, $K_{\mathrm{n}}$, and the specific friction cutting force, $K_{\mathrm{f}}$, can be determined as follows:

$$
\begin{aligned}
K_{\mathrm{n}} & =N / A_{\mathrm{c}} \\
K_{\mathrm{f}} & =P / N,
\end{aligned}
$$

where $N$ and $P$ are the force components normal and parallel to the rake face of the tool, and $A_{\mathrm{c}}$ is the uncut chip area. Following Nair et al. (1999), after the first revolution the uncut chip area the uncut chip area, $A_{\mathrm{c}}$, can be found using:

$$
\begin{aligned}
& A_{\mathrm{c}}=f d-f\left(r_{\mathrm{n}}-\sqrt{r_{\mathrm{n}}^{2}-f^{2} / 4}\right) / 2, r_{\mathrm{n}} \geq f / 2 \\
& A_{\mathrm{c}}=f d, r_{\mathrm{n}}<f / 2,
\end{aligned}
$$

where $f$ is the feed rate, $d$ is the depth of cut and $r_{\mathrm{n}}$ is the tool nose radius. On the other hand, according to Stephenson and Bandyopadhyay (1995), in bar turning, the tangential, axial and radial cutting force are given as follows:

$$
\begin{aligned}
& F_{\mathrm{t}}=K_{\mathrm{n}} A_{\mathrm{c}}\left[\cos \alpha_{\mathrm{b}} \cos \alpha+K_{\mathrm{f}}\left(\cos \gamma_{\mathrm{Le}} \sin \alpha+\sin \gamma_{\mathrm{Le}} \sin \alpha_{\mathrm{b}}\right)\right] \\
& F_{\mathrm{a}}=K_{\mathrm{n}} A_{\mathrm{c}}\left(-\cos \alpha_{\mathrm{b}} \sin \alpha+K_{\mathrm{f}} \cos \gamma_{\mathrm{Le}} \cos \alpha\right) \\
& F_{\mathrm{r}}=K_{\mathrm{n}} A_{\mathrm{c}}\left(-\sin \alpha_{\mathrm{b}}+K_{\mathrm{f}} \sin \gamma_{\mathrm{Le}} \cos \alpha_{\mathrm{b}}\right),
\end{aligned}
$$


where $\alpha_{\mathrm{b}}$ is the back rake angle; $\alpha$ is the normal rake angle, $\gamma_{\mathrm{Le}}$ is the effective lead angle. In particular, the effective lead angle, $\gamma_{\text {Le }}$, determines the direction of the friction force. For relatively deep cuts in which the ratio of the depth of cut to the tool nose radius is large (e.g. $>5$ ), the effective lead angle is approximately equal to the lead angle $\gamma_{\mathrm{L}}$. When $-5<\gamma_{\mathrm{L}}<45^{\circ}, 0.5<d<5.0 \mathrm{~mm}, 0.1<f<1.0 \mathrm{~mm}$, $0.4<r_{\mathrm{n}}<4.4 \mathrm{~mm}$ and $r_{\mathrm{n}}>f$, the effective lead angle $\gamma_{\mathrm{Le}}$ satisfies the following equation:

$$
\tan \gamma_{\mathrm{Le}}=0.5053 \tan \gamma_{\mathrm{L}}+1.0473 \frac{f}{r_{\mathrm{n}}}+0.4654 \frac{r_{\mathrm{n}}}{d} .
$$

Based on the tangential force, the radial force and the tool geometry parameters $\alpha$, $\alpha_{\mathrm{b}}, \gamma_{\mathrm{LE}}$, and $A_{\mathrm{c}}$, the specific cutting force coefficients, $K_{\mathrm{n}}$ and $K_{\mathrm{f}}$, can be estimated using equations (17) and (18). Furthermore, using equation (19), the radial force, $F_{\mathrm{r}}$, can then be found.

In summary, the cutting force-induced error is estimated in two stages. In the first stage, the cutting forces are estimated based on the cutting conditions and the motor currents using two RBF ANNs. Note that equations (15-20) are never used explicitly as they are approximated by the ANNs. In the second stage, the cutting force-induced error is estimated based on the cutting forces using another RBF ANN. This is shown in figure 9.

\subsection{Workpiece error compensation}

According to the literature, from an implementation point of view, there are two ways to compensate the workpiece errors. One is the hardware approach, which uses

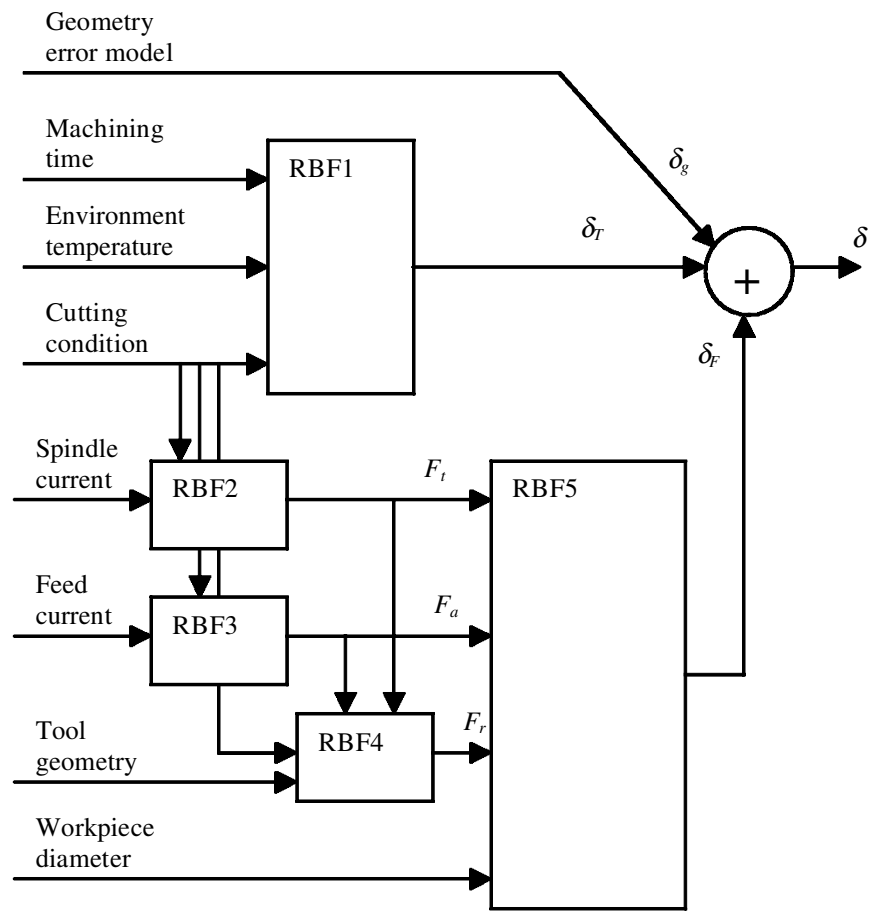

Figure 9. The workpiece error prediction model. 
additional hardware such as a hydraulic actuator. The other uses only the software by overwriting the $\mathrm{CNC}$ codes. From a control point of view, the compensation methods include open-loop compensation and closed-loop compensation. The former uses a predetermined compensation strategy while the later depends on the on-line measurement. Here, a software closed-loop compensation method is used.

The workpiece error compensation is done in two steps. First, a number of experiments were conducted to acquire data for training the RBF ANNs. Note that during the training, both the motor currents and the cutting forces are needed to train the RBF ANNs. With the trained RBF ANNs, given a set of tool geometry and cutting condition, the workpiece error can be estimated. Accordingly, the CNC codes are modified to compensate the error. The modification has a resolution of $1 \mu \mathrm{m}$ in the direction of the depth of cut. The experimental results are presented below.

\section{Experimental results and discussions}

During the experiments, various sensors and measurement devices were used. First, the spindle and feed motor currents were measured using a pair of PCB Hall effect current transducers. The signals were first passed through a low-pass filter, and then recorded by a tape recorder. The cutting forces were measured using a Kistler dynamometer. Note that the cutting forces were used only as the mean to train the ANNs and were not needed after the training.

A total of 26 cutting tests were conducted under various cutting conditions as shown in table 1 . The variations of the cutting conditions include the following.

- Cutting speed $v$, two levels: 2.5 and $4.0 \mathrm{~mm} / \mathrm{s}$.

- Feed rate $f$, three levels: $0.1,0.15$ and $2.0 \mathrm{~mm} / \mathrm{rev}$.

- Depth of cut $d$, two levels: 0.5 and $1.0 \mathrm{~mm}$.

- Workpiece diameter $D$, three levels: 30,40 and $50 \mathrm{~mm}$.

- Tool nose radius $R$, two levels: 0.4 and $0.8 \mathrm{~mm}$.

- Coolant, two levels: with (D) and without coolant (F).

- Workpiece material: two levels: mild steel (S) and aluminium (A).

The first 21 cutting tests were used to establish the models for thermal-induced error and cutting force-induced error (i.e., training the hybrid RBF neural networks). The rest of the cutting tests were used to verify the presented method.

Figure 10 shows a comparison between the measured cutting force and the estimated cutting force. It is seen that the difference between the measured and the estimated tangential cutting forces is $<10 \%$ in all cases. The difference between the measured and the estimated axial cutting forces is $<5 \%$ in all cases. For the radial forces, however, the difference is significant ranging from 10 to $25 \%$. This may be attributed to the fact that the estimated radial force depends strongly on the lead angle, which is dependent on the tool wear and the workpiece material. As mentioned earlier, the use of motor current to estimate the cutting force is advantageous . In fact, a typical current sensor costs only about US\$65 and requires little additional hardware. In addition, it is durable, flexible and easy to install. Therefore, the current sensors have a great potential for shop floor applications.

Using the presented workpiece error prediction model to compensate the workpiece error results in a significant improvement on dimension accuracy. Figure 11 


\begin{tabular}{|c|c|c|c|c|c|c|c|}
\hline \multirow{2}{*}{$\begin{array}{l}\text { Experiment } \\
\text { no. }\end{array}$} & \multicolumn{7}{|c|}{ Cutting condition } \\
\hline & $\nu(\mathrm{m} / \mathrm{s})$ & $f(\mathrm{~mm} / \mathrm{rev})$ & $d(\mathrm{~mm})$ & $D(\mathrm{~mm})$ & Tool nose & Coolant & Material \\
\hline 1 & 2.50 & 0.10 & 0.50 & 50 & 0.4 & D & $\mathrm{S}$ \\
\hline 2 & 4.00 & 0.10 & 0.50 & 40 & 0.4 & D & $\mathrm{S}$ \\
\hline 3 & 4.00 & 0.20 & 1.00 & 30 & 0.4 & D & $\mathrm{S}$ \\
\hline 4 & 2.50 & 0.10 & 0.50 & 50 & 0.8 & $\mathrm{~F}$ & $\mathrm{~S}$ \\
\hline 5 & 4.00 & 0.15 & 1.00 & 40 & 0.8 & $\mathrm{~F}$ & $\mathrm{~S}$ \\
\hline 6 & 2.50 & 0.20 & 1.00 & 30 & 0.8 & $\mathrm{~F}$ & $\mathrm{~S}$ \\
\hline 7 & 2.50 & 0.10 & 1.00 & 50 & 0.4 & $\mathrm{~F}$ & A \\
\hline 8 & 4.00 & 0.15 & 1.00 & 40 & 0.4 & $\mathrm{~F}$ & A \\
\hline 9 & 2.50 & 0.20 & 1.00 & 30 & 0.4 & $\mathrm{~F}$ & A \\
\hline 10 & 4.00 & 0.20 & 1.00 & 50 & 0.8 & $\mathrm{~F}$ & A \\
\hline 11 & 2.50 & 0.15 & 1.00 & 40 & 0.8 & $\mathrm{~F}$ & A \\
\hline 12 & 2.50 & 0.10 & 0.50 & 30 & 0.8 & $\mathrm{~F}$ & A \\
\hline 13 & 4.00 & 0.20 & 1.00 & 50 & 0.4 & D & A \\
\hline 14 & 2.50 & 0.15 & 1.00 & 40 & 0.4 & $\mathrm{D}$ & A \\
\hline 15 & 2.50 & 0.10 & 1.00 & 30 & 0.4 & $\mathrm{D}$ & A \\
\hline 16 & 4.00 & 0.20 & 1.00 & 50 & 0.8 & D & A \\
\hline 17 & 2.50 & 0.15 & 1.00 & 40 & 0.8 & $\mathrm{D}$ & A \\
\hline 18 & 2.50 & 0.10 & 1.00 & 30 & 0.8 & $\mathrm{D}$ & A \\
\hline 19 & 4.00 & 0.20 & 1.00 & 50 & 0.8 & $\mathrm{D}$ & $\mathrm{S}$ \\
\hline 20 & 2.50 & 0.15 & 1.00 & 40 & 0.8 & $\mathrm{D}$ & $\mathrm{S}$ \\
\hline 21 & 2.50 & 0.20 & 0.50 & 30 & 0.8 & D & $\mathrm{S}$ \\
\hline $\mathrm{T} 1$ & 2.50 & 0.10 & 1.00 & 45 & 0.4 & $\mathrm{~F}$ & $\mathrm{~S}$ \\
\hline T2 & 4.00 & 0.20 & 1.00 & 35 & 0.8 & D & $\mathrm{S}$ \\
\hline T3 & 4.00 & 0.15 & 1.00 & 45 & 0.8 & $\mathrm{~F}$ & $\mathrm{~S}$ \\
\hline $\mathrm{T} 4$ & 4.00 & 0.15 & 0.50 & 35 & 0.4 & $\mathrm{D}$ & A \\
\hline T5 & 2.50 & 0.15 & 1.00 & 45 & 0.4 & $\mathrm{~F}$ & A \\
\hline
\end{tabular}

Table 1. Training and test examples.

shows five sets of experiment results (experiment nos T1, T2, T3, T4 and T5 in table 1). From figure 11, following observation s can be made.

- The thermal-induced error is a constant independent on the workpiece length and its effect is usually small.

- The cutting force-induced error increases with respect to the increase of the length of the workpiece. This is because during the cutting tests, the tailstock was not used. Hence, there were larger deflections when the cutting approached the end of the workpiece. Incidentally, the cutting force-induced error was in the opposite direction of the geometry in the experiment, and hence resulted in reduced total workpiece error. In practice, however, it is possible that all the three error components could be in the same direction resulting large total workpiece error.

- The estimated workpiece error is very close to the actual workpiece error obtained from FTS-Q sensing. In fact, the average error between the estimated workpiece error and the actual workpiece error is $<5 \%$. This indicates that the presented model is very effective.

- The compensation can reduce the workpiece error to within $\pm 8 \mu \mathrm{m}$. In comparison, without the compensation, the workpiece errors were about $\pm 14 \mu \mathrm{m}$. 


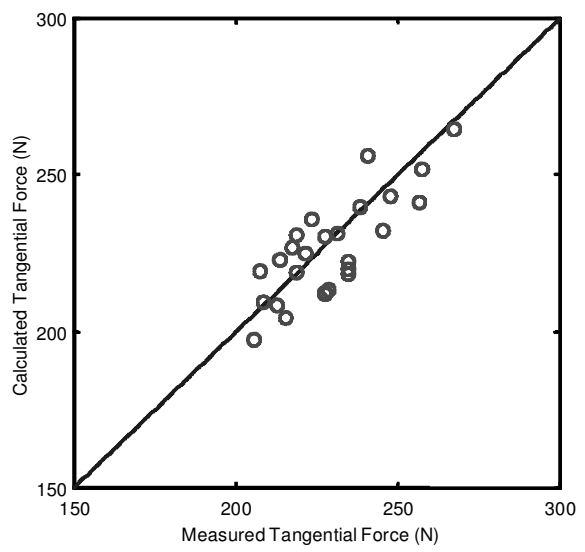

(a)

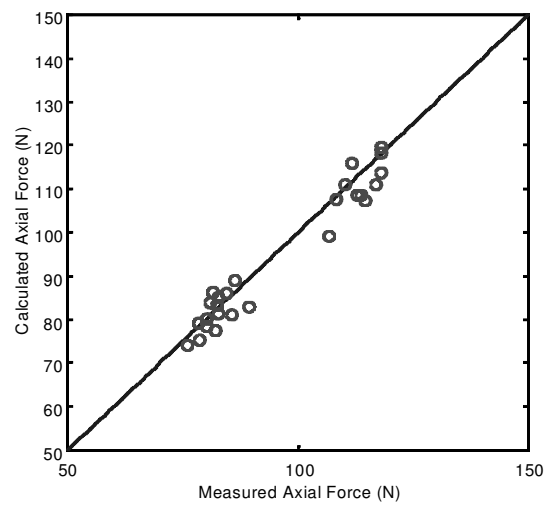

(b)

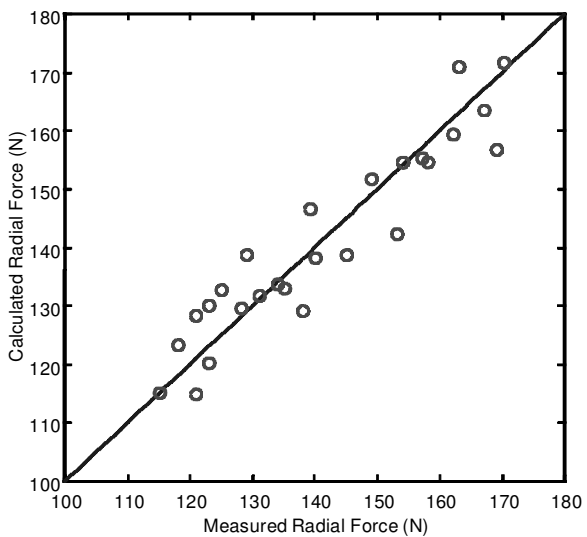

(c)

Figure 10. Comparison of actual and estimated cutting forces. Cutting speed: 2.5, $4(\mathrm{~m} / \mathrm{s})$; feed rate: $0.1,0.15,0.2(\mathrm{~mm} / \mathrm{rev}$.$) ; the depth of cut: 0.5,1(\mathrm{~mm})$; tool geometry: $\gamma_{\mathrm{L}}=-3^{\circ}$, $\alpha=-3.5^{\circ}, \alpha_{\mathrm{b}}=-7^{\circ}$; nose radius of tools: $r_{\mathrm{n}}=0.4,0.8 \mathrm{~mm}$; workpiece: mild steel. 


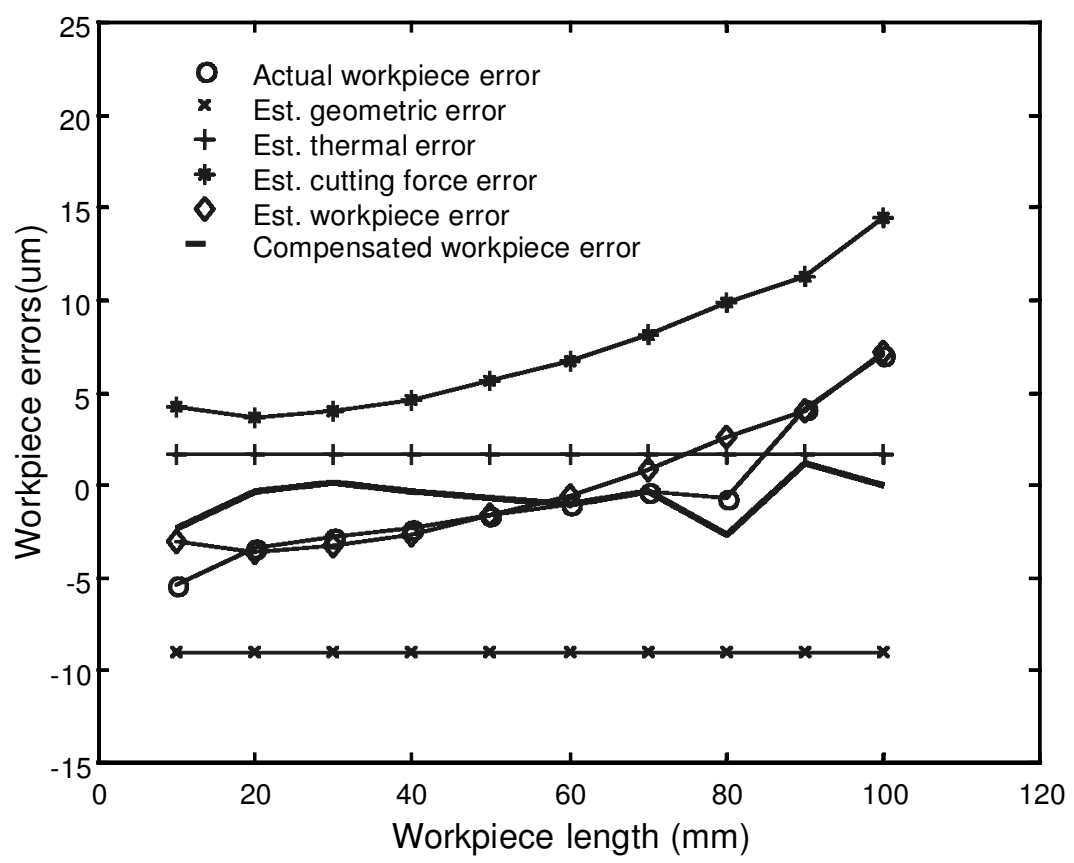

\section{1 (a) Test 1}

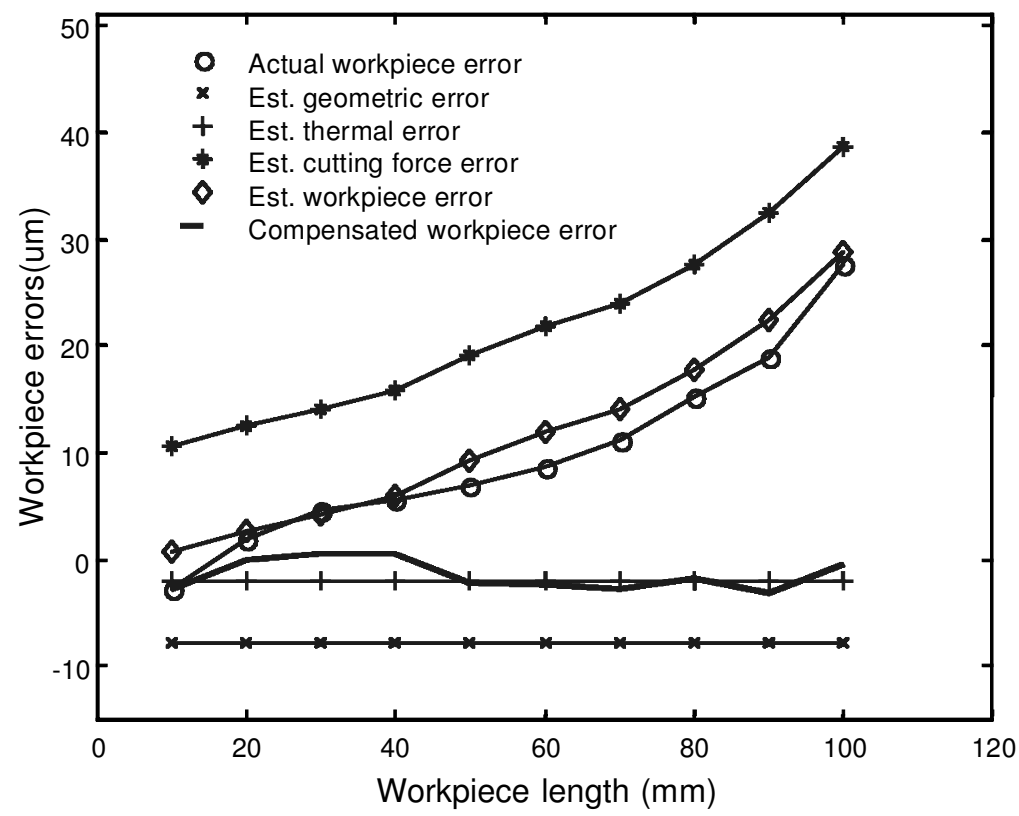

11(b) Test 2 


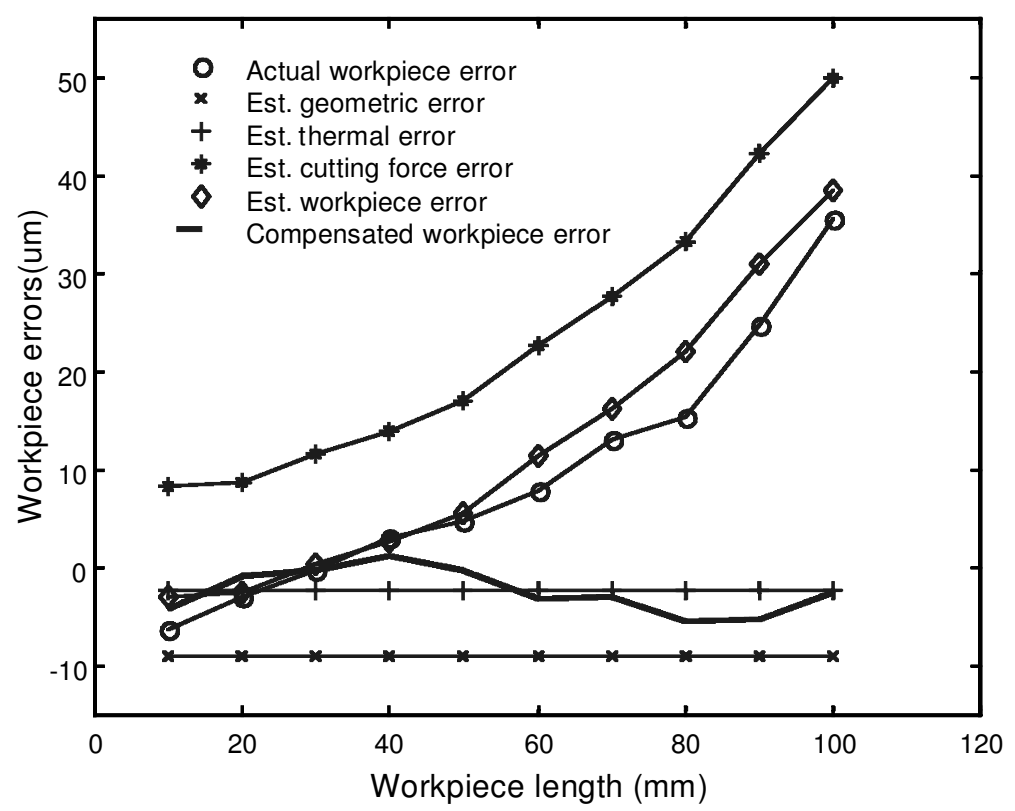

11 (c) Test 3

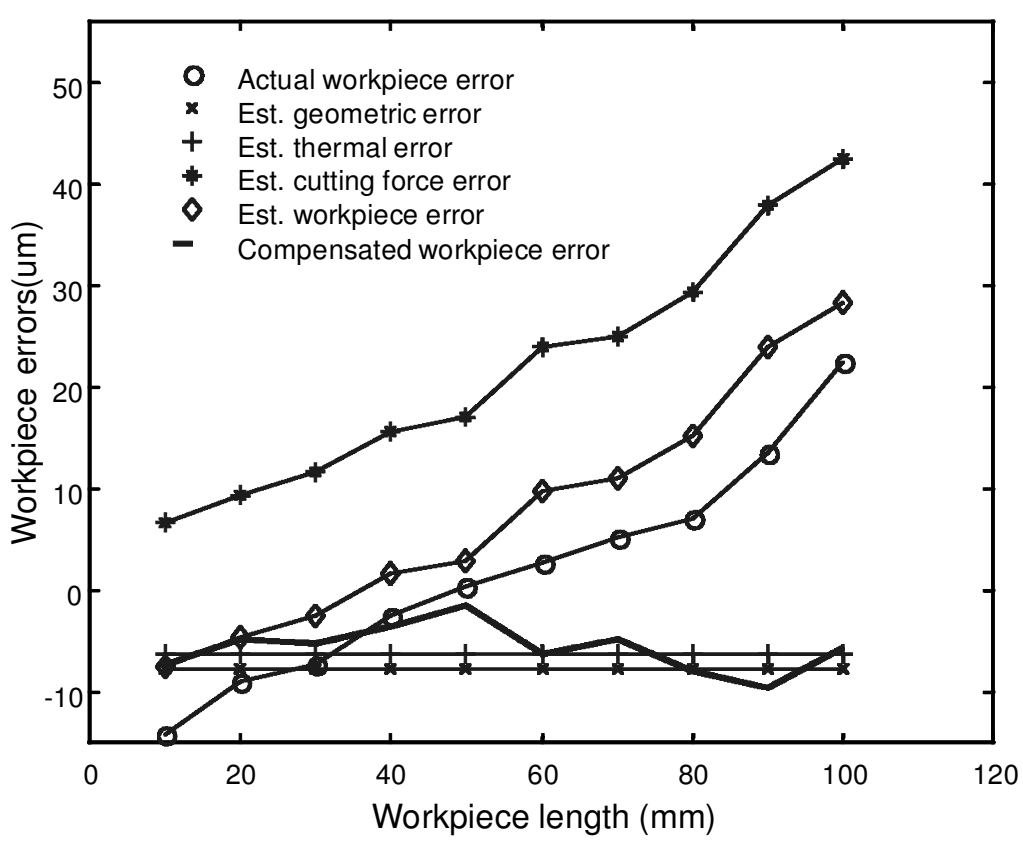

11 (d) Test 4 


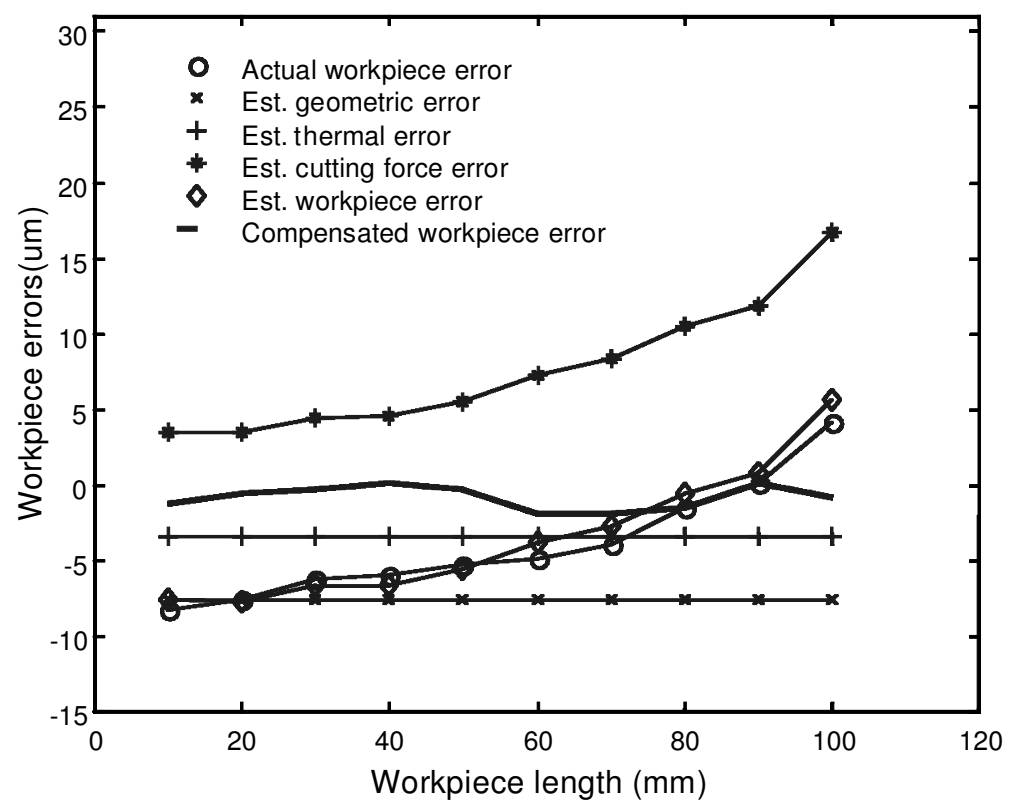

(e)

Figure $11-$ concluded. Experimental results.

This indicates that the presented error compensation method is indeed very effective.

\section{Conclusions}

Based on the experimental results and discussions above, following conclusions can be drawn.

- In turning, the workpiece errors may consist of two parts: the error related to the machine tool (including geometric error of the machine tool and the thermal-induced error of the machine tool), and the error related to the cutting.

- The workpiece error can be accurately measured using the presented FTS-Q sensing system. Its measurement error is $<5 \%$.

- To measure and, hence, compensate the workpiece error on-line, it is necessary to develop models for each error component. The geometric error can be modelled using a simple linear regression model based on the experimental data without machining. The thermal-induced error is dependent on cutting conditions and machining time. It can be predicted by a RBF ANN. The cutting force-induced error is dependent on the cutting forces and it can be predicted using a two-stage RBF ANN based on the spindle motor current and the feed motor current. In the first stage, two RBF ANNs and an analytical model are used to estimate the cutting forces. Based on the estimated cutting 
forces, in the second stage, another RBF neural network is used to model the cutting force-induced error.

- The workpiece error can be compensated for by simply modifying the CNC codes (software compensation). Based on the experimental testing, the presented model can reduce the workpiece error to within $\pm 5 \mu \mathrm{m}$ from $\pm 14 \mu \mathrm{m}$.

- The presented error compensation method uses only the spindle motor current and the feed motor current, as well as software implementation. Hence, its cost would be low and has a great potential for shop floor applications.

\section{Acknowledgement}

The research was partially supported by the City University of Hong Kong, DAG Grant No. 7100063.

\section{Appendix A: Brief description of the hybrid RBF neural network}

The radial basis function ( $\mathrm{RBF}$ ) neural network is one of the most commonly used artificial neural networks (ANNs). Its theory and applications can be found in several monographs and papers, such as Haykin (1994). Briefly, a RBF neural network is a non-linear mapping function that transforms a set of $n$-dimensional inputs to a set of $m$-dimensional outputs (figure 5). The non-linear transformation is controlled by a set of $m$ base functions. Each base function is characterized by a centre vector, $c_{j}=\left[c_{j 1}, c_{j 2}, \ldots, c_{j m}\right]$, and a radius vector $\boldsymbol{r}_{j}=\left[r_{j 1}, r_{j 2}, \ldots, r_{j m}\right]$. A typical base function is:

$$
h_{j}(\mathbf{x})=\exp \left[-\sum_{k=1}^{n} \frac{\left(x_{k}-c_{j k}\right)^{2}}{r_{j k}^{2}}\right],
$$

where $\mathbf{x}=\left[x_{1}, x_{2}, \ldots, x_{n}\right]$ represents a training sample. From equation (A.1), it is seen that the output of the base functions will have the largest responds to the inputs nearest to the centre $\boldsymbol{c}_{j}$. In addition, the radius $\boldsymbol{r}_{j}$ can be considered as a weighting function. Based on the base functions, the output of the RBF neural network, $f(\mathbf{x})$, is determined by a linear regression:

$$
f(\mathrm{x})=\sum_{j=1}^{m} w_{j} h_{j}(\mathrm{x}),
$$

where $w_{j}$ are weighting factors.

To build a RBF neural network is to determine: (1) the number of nodes of the network; (2) the Gaussian centre $\boldsymbol{c}_{j}$; (3) the radius $\boldsymbol{r}_{j}$; and (4) the output-layer weighting factors $w_{j}$. The first three problems are often solved together since the number of nodes are correlated to the Gaussion centre $\boldsymbol{c}_{j}$ and the radius $\boldsymbol{r}_{j}$. According to the literature, various algorithms have been developed (Broomhead and Lowe 1988, Poggio and Girosi 1990, Chen et al. 1991, Cheng and Lin 1994). To determine the weighting factor $w_{j}$ is relatively easy. It can be done using conventional statistical approaches such as the least mean squares and pseudo-inverse matrix.

In the presented research, the method by Kubat (1998) and Orr (2000) is used. Kubat (1998) first proposed using a decision tree to build a RBF neural network. First, a decision tree is built using algorithms. Then, each node of the decision tree is 
converted to a node of the RBF neural network. In this way, the structure of RBF can be determined and consequently the RBF network can be built. Orr (2000) modified this approach by combining a regression decision tree and RBF neural network with a complexity control parameter. It consists of the following steps.

- Generating a regression tree. Given a set of training data, $\left\{\mathrm{x}_{i}\right\}_{i=1}^{p}$, a decision tree can be obtained by the regression tree building methods. Note that each node of the tree represents a hyper-rectangle in the sample space. The centres of these hyper-rectangles, $\boldsymbol{c}$, can also be calculated.

- Transforming the nodes of the decision tree into RBFs. To transform a hyperrectangle into a Gaussian $\mathrm{RBF}$, the centre of the hyper-rectangle, $\boldsymbol{c}$, is taken as the RBF centre, and its size (half-width), scaled by a control parameter $\alpha$, is used to form the RBF radius, $\boldsymbol{r}$.

- Selecting a subset of RBFs. In general, if a RBF contains all the nodes of the decision tree, the RBF could become oversensitive. To minimize the effect of this problem, it is necessary to use a backward elimination. The backward elimination is based on how many training samples that a node is associated to. The nodes that associate only a few training samples would be eliminated.

- Calculating the weighting factors. Using the same training data $\left\{\mathrm{x}_{i}\right\}_{i=1}^{p}$, the weighting factor, $w_{j}$, can be determined by:

$$
\mathrm{w}=\left(\mathrm{H}^{\mathrm{T}} \mathrm{H}\right)^{-1} \mathrm{H}^{\mathrm{T}} \mathrm{y}
$$

where $\mathrm{H}=\left\{h_{j}\left(\mathrm{x}_{i}\right)\right\}$ and $\mathbf{y}=\left[\begin{array}{llll}y_{1} & y_{2} & \cdots & y_{p}\end{array}\right]^{\mathrm{T}}$ are the outputs of the training data.

- Evaluating the RBFs. To determine when the training of the RBF is completed, two criteria can be used. These are the generalized cross-validation (GCV) and Bayesian information criterion (BIC).

It should be pointed out that in the Orr method, there are two important control parameters: one is used to control the depth of the regression tree and the other is used to control the ratio of the number of hyper-rectangles and the number of nodes in the RBF. Additional calculations are needed to acquire these parameters.

\section{References}

Altintas, Y. and Dong, C., 1996, Design and analysis of a modular CNC system for machining control and monitoring. Modeling of Machine Tools: Accuracy, Dynamics, and Control American Society of Mechanical Engineers, Vol. 45 (New York: ASME), pp. 199-208.

Asao, T., Mizugaki, Y. and Sakamoto, M., 1992, Precision turning by means of a simplified predictive function of workpiece error. Annals of the CIRP, 41, 447-451.

Bryan, J., 1990, International status of thermal error research. Annals CIRP, 39, 645-656.

Broomhead, D. and Lowe, D., 1988, Multivariate functional interpolation and adaptive networks. Complex Systems, 2, 321-355.

Chang, Y., Lee, K. and Chuang, H., 1995, Cutting force measurement of spindle motor. Journal of Control Systems and Technology, 3, 145-152.

Chen, S., Cowan, C. and Grant, P., 1991, Orthogonal least squares learning algorithm for radial basis function networks. IEEE Transactions on Neural Networks, 2, 302-309. 
Chen, S., Ulsoy, A. G. and Koren, Y., 1998, Error source diagnostic using a turning process simulator. Transactions of ASME, Journal of Manufacturing Science and Engineering, 120, 409-416.

Endres, W., Sutherland, J., Devor, R. and Kapoor, S., 1992, A dynamic model of the cutting force system in the turning process. Monitoring and Control for Manufacturing Process (New York: ASME PED, ASME), vol. 44, pp. 193-212.

Haber, R., Peres, C., Alique, A., Salvador, G., Carlos, A. and Alique, J., 1998, Toward intelligent machining: hierarchical fuzzy control for the end milling process. IEEE Transactions on Control Systems Technology, 6, 188-199.

Hay kin, S., 1994, Neural Networks, A Comprehensive Foundation (New York: Macmillan).

KIM, T. and KIM, J., 1996, Adaptive cutting force control for a machining center by using indirect cutting force measurements. International Journal of Machine Tools and Manufacture, 36, 925-937.

Kubat, M., 1998, Decision trees can initialize radial-basis function networks. IEEE Transaction on Neural Networks, 9, 813-824.

Kops, L., Gould, M. and Mizr aCH, M., 1993, Improved analysis of the workpiece Accuracy in turning based on the emerging diameter. ASME Journal of Engineering for Industry, 115, 253-257.

Kops, L., Gould, M. and Mizrach, M. 1994, Search for equilibrium between workpiece deflection and depth of cut: key to predict compensation for deflection in turning. Manufacturing Science and Engineering, ASME PED vol. 68, 819-825.

Lee, J., Choi, D., KIm, J. and Chu, C., 1995, Real-time tool breakage monitoring for NC milling process. CIRP Annals, 44, 59-62.

LI, X., 2001, Real time prediction of workpiece errors for a CNC turning centre, Part 2. Modelling and estimation of thermally induced errors. International Journal of Advances in Manufacturing Technology (in press).

Li, X., Venuvinod, P. K. and Chen, M. K., 2000, Feed cutting force estimation from the current measurement with hybrid learning. International Journal of Advances in Manufacturing Technology, 16, 859-862.

LiU, Z. Q. and Venuvinod, P. K., 1999, Error compensation in CNC turning solely from dimensional measurements of previously machined parts. Annals of the CIRP, 48, 429432.

Nair, R., Danai, K. and Malkin, S., 1991, Turning process identification through force transients. Automation of manufacturing processes. 1991 ASME WAM, Dallas, TX, PED vol. 52, pp. 59-66.

OrR, M. J. L., 2000, Combining regression trees and RBFs. International Journal of Neural Systems (in press).

Ostafiev, V., Masol, I. and Tiмchiк, G., 1991, Multiparameters intelligent monitoring system for turning. Proceedings of the 1991 SME International Conference, Las Vegas, NV, pp. 296-300.

Ostafiev, V. and Venuvinod, P. K. 1997, A new electromagnetic contact sensing technique for enhancing machining accuracy. IMECE-97, ASME, pp. 245-252.

Phan, A., Cloutier, G. and René Mayer, J., 1999, An investigation into diameter error modeling in bar turning. 1999 NSF Design \& Manufacturing Grantees Conference [distributed material].

Poggio, T. and Girosi, F., 1990, Regularization algorithms for learning that are equivalent to multilayer networks. Science, 247, 987-982.

Stein, J., Colvin, D., Clever, G. and WAng, C., 1986, Evaluation of de servo machine tool feed drives as force sensors. Transactions of ASME, Journal of Dynamic Systems Measurement and Control, 108, 279-288.

Stein, J. and HuH, K., 1991, A design procedure for model-based monitoring systems: cutting force measurement as a case study. Control of Manufacturing Processes, ASME DSC Vol. 28/PED Vol. 52, 45-57.

Stephenson, D. and BAndyopadhy ay, P., 1995, Process independent force characterization for machining simulation. Concurrent Product and Process Engineering, ASME MED Vol. 1/DE Vol. 85, 15-36.

Veldhuis, S. and Elbestawi, M., 1995, A strategy for the compensation of errors in five-axis machining. Annals CIRP, 44, 373-377. 
YANG, S., YUAN, J. and Ni, J., 1997, Real-time cutting force induced error compensation on a turning center. International Journal of Machine Tools and Manufacture, 37, 1597-1610.

YUAN, J. and NI, J., 1998, The real-time error compensation technique for CNC machining systems. Mechatronics, 8, 359-380.

Zhang, G., Yerramareddy, S., Lee, S. and Lu, S., 1991, Simulation of intermittent turning processes. ASME Journal of Engineering in Industry, 113, 485-466. 